\title{
МЕТОД ИДЕНТИФИКАЦИИ ЗАКОНОМЕРНОСТЕЙ
}

Мазуркин Петр Матвеевич

д-р техн. наук, профессор Поволжского государственного технологического университета, г. Йошкар-Ола

E-mail: kaf_po@mail.ru

\section{METHOD OF IDENTIFICATION OF LAWS}

Mazurkin Peter Matveevich

Dr. Sc. , Professor of the Volga State Technological University, Yoshkar-Ola

E-mail: kaf_po@mail.ru

\section{АННОТАЦИЯ}

Идентификация закономерностей представляется как процесс решения задачи статистического моделирования асимметричными вейвлет-сигналами по рядам измерений. Показана методология идентификации устойчивых законов любого процесса, включая и экономические, волновыми функциями с переменными амплитудой и частотой колебания. Статья дана как решение 23-ой проблемы Гильберта универсальным алгебраическим уравнением в общей форме, предложенной автором, и тем самым доказана гипотеза Декарта.

\section{ABSTRACT}

Identifying laws it is represented as a process of solving the problem of statistical modeling asymmetric wavelet signals for measurement series. It is shown that the methodology of identification of stable laws of any process, including economic, wave functions with variable-governmental amplitude and frequency fluctuations. Article is given as a solution to the 23rd pro-Hilbert problem universal algebraic equation in the general form, proposed by the author, and thus proved the hypothesis of Descartes.

Ключевые слова: статистические данные, табличные модели, закономерности, вейвлет-сигналы, идентификация, методология

Keywords: statistics, spreadsheet models, patterns, wavelet signals, identification, methodology 
Введение. На основе первичности практики над теорией [1] предложен метод идентификации закономерностей на основе устойчивых законов, прежде всего обобщенной формулы в виде уравнения биотехнического закона [2-10], относительно заданных статистических данных в табличной форме. Это позволяет отказаться от теории «нормальных» распределений Гаусса-Лапласа и создать независимую от отраслей экономики, науки и техники теорию статистического моделирования. Действительно нормальными становятся волновые распределения (вейвлет-сигналы) параметров изучаемой системы.

Итак, пусть имеются статистические данные, к которым также можно отнести и данные, полученные исследователем в ходе активных или пассивных экспериментов. Причем под словами «статистические данные» следует понимать не только цифровую информацию, но и ряды единичных количественных сведений об объектах или субъектах, явлениях или процессах.

Статистика зародилась для хозяйственного учета, а затем стала использоваться для потребностей государственного управления. Ныне статистика охватывает не только все нужды человека и общества, но и потребности других живых существ и их сообществ, прежде всего животных и растений, а также природных, природно-техногенных и техногенных (технических) объектов.

Исследователю придется соблюдать одно требование - это достоверность исходных данных (для экономистов также и полнота исходных данных) в формируемых (или принимаемых по отчетам и публикациям других авторов) табличных моделях. Этот входной контроль должен быть выполнен, чтобы в процессе статистического моделирования не возникали эвристические противоречия и подозрения о недоброкачественности исходных данных.

Освоение предлагаемой методологии статистического моделирования позволит изменить традиционное планирование эксперимента, в частности, определение периодичности измерений и продолжительности наблюдений. При выборе закономерности нужно отказаться от полиноминальной (квадратичной, кубической и другой) модели регрессионного анализа. Статистическая обработка результатов эксперимента будет выполняться по устойчивым закономер- 
ностям с волновыми составляющими. Проверка адекватности статистической модели выполняется автоматически по коэффициенту корреляции. Интерпретация результатов эксперимента будет точнее по параметрам закономерности.

Общие сведения. Проблема развития методов вариационного исчисления (23-я проблема Гильберта) до сих пор не решена, хотя в этом направлении математики много сделано самим Гильбертом. Математическую деятельность Давида Гильберта условно можно разделить на две разнонаправленные научные концепции (векторы понимания):

а) интегральные уравнения и физика (включает все науки: астрономия, биология и экология, эконометрика, техника и технология и др.);

б) алгебраические числовые поля и инварианты.

Два этих вектора понимания (концепции) вместе объединяет теория аксиоматики Гильберта. Таким образом, аксиоматика лежит в основе обоих подходов - от математика к физику-теоретику (первый вектор) и, наоборот, от экспериментатора к математику (этот подход известен и в экономике [1]).

Монарный подход математика-теоретика к развитию абстрактной математики мы не рассматриваем. На многих примерах (более 100 тысяч) статистического моделирования убедились в том, что решение 23-й проблемы Гильберта находится не в математике или физике в отдельности, а именно в переходе от экспериментальной физики (измерения в физике, астрономии, биологии и экологии, социологии и эконометрике, технике и технологии) к известным и новым искомым методам прикладной математики.

Ключом к пониманию классическими математиками предлагаемой нами методологии идентификации инвариантов по реальным алгебраическим числовым полям, как результатам многофакторных физических измерений.

Но у нас инвариантами являются гладкие и явно нелинейные устойчивые законы [2-10], включая и волновые функции, применимые к вещественным числовым полям (статистическим выборкам). При этом каждый физический фактор в принятом множестве, например, для описания поведения системы, обособленно может приниматься за объясняющую переменную. 
Методология идентификации закономерностей. Подробнее отдельные особенности предложенной методологии даны в [2-10]. Методика и технология пользования программной средой типа CurveExpert показана в [6].

Идентификация по числовому полю (табличной модели) включает этапы:

1) эвристическая идентификация сути изучаемого явления или процесса по результатам измерений и анализа табличной модели (априорная информация) и осмысления в ходе выявления конструкции закономерности по её аддитивным составляющим (апостериорная информация);

2) структурная идентификация однофакторной математической функции в общей модели как суммы инвариантов из вейвлет-сигналов;

3) параметрическая идентификация наращиваемой по инвариантам структуры статистической закономерности в среде CurveExpert-1.40.

Идентификация эвристическая. Она предполагает знание предмета исследования, поэтому здесь важен переход от физики к математике, а не наоборот. Главное эвристическое значение приобретает заданное числовое поле, как правило, оформляемое по результатам измерений в виде таблицы. В ней неявно заключен весь содержательный смысл (эвристика) и понимание (концепция) автора измерений (или составителя общей таблицы по результатам многих авторов) по глубинному смыслу эксперимента (или обобщения данных).

Критерием (количественной мерой) процесса идентификации становится погрешность измерений при составлении числового поля, т.е. таблицы исходные данных, в том числе и для других этапов идентификации. Например, простые числа и их ряды не имеют погрешности измерения: они абсолютно достоверны, добротны и надежны. А другие виды исходных данных имеют разную погрешность. Таким образом, математические числовые объекты - наилучшие для идентификации законов-инвариантов.

На втором месте находятся прецизионные измерения в астрономии, физике, технике и технологии, других областях науки.

На третье место встают биологические объекты, и их поведение имеет четко проявляющийся колебательный характер, поэтому линейные и линеаризо- 
ванные модели биологам вообще не подходят.

Самые высокие погрешности имеют социально-экономические измерения из-за их высокой субъективности содержательных представлений о поведении общественных, природных и природно-антропогенных объектов.

Мы давно отказались от методов аппроксимации и поэтому вообще не применяем программы статистики из Excel при подборе уравнений регрессии. В идентификации первый этап (вариация функций) из теории аппроксимации исключается, - устойчивые законы заранее заданы как инварианты. Из них как из кирпичиков можно создавать различные математические конструкты.

Использование в конкретном примере статистического (вероятностного) моделирования того или иного устойчивого закона (по таблице 1 их всего семь) требует предварительного эвристического осмысления задачи моделирования.

Таким образом, на эвристическом уровне 23-я проблема Гильберта нами пока недостаточно формализована. Но она была полностью решена на структурном и параметрическом уровнях анализа и синтеза. Вариация функций сводится к осознанному отбору устойчивых законов и конструированию на их основе адекватных данным устойчивых волновых закономерностей. В будущем предполагается автоматизированное конструирование однофакторных моделей для любых табличных данных (числовых матриц) по многофакторным измерениям. Для этого нужно создать программную среду по нашим сценариям.

Идентификация структурная. Декарт предполагал существование одного-единственного алгебраического уравнения, пригодного как однозначный вариант решения для любых типов интегральных уравнений. Гильберт мечтал об инвариантах, из которых как из кирпичиков будет собираться это универсальное уравнение. Наши универсальные инварианты даны в таблице 1.

Они сгруппированы по принципу «от простого к сложному».

По сути, фрагменты и сам биотехнический закон являются «кирпичиками Гильберта» для построения, в ходе процесса структурно-параметрической идентификации, аддитивной или мультипликативной конструкции искомой статистической детерминированной или волновой модели. 
Математические конструкты (исходные инварианты) для составления модели

\begin{tabular}{|c|c|}
\hline $\begin{array}{c}\text { Фрагменты без предыстории } \\
\text { изучаемого явления или процесса }\end{array}$ & $\begin{array}{c}\text { Фрагменты с предысторией } \\
\text { изучаемого явления или процесса }\end{array}$ \\
\hline $\begin{array}{l}y=a x-\text { закон линейного роста или спада } \\
\text { (при отрицательном знаке перед правой сто- } \\
\text { роной формулы линейного изменения) }\end{array}$ & $\begin{array}{l}y=a-\text { закон не влияния принятой объяс- } \\
\text { няющей переменной на показатель, который } \\
\text { имеет собственную предысторию значений }\end{array}$ \\
\hline $\begin{array}{l}y=a x^{b}-\text { закон показательного роста (закон } \\
\text { показательной гибели } y=a x^{-b} \text { не является } \\
\text { устойчивым, из-за бесконечности показателя } \\
\text { при нулевом значении объясняющей пере- } \\
\text { менной) }\end{array}$ & $\begin{array}{l}y=a \exp ( \pm c x)-\text { закон Лапласа в математике } \\
\text { (Ципфа в биологии, Парето в экономике, } \\
\text { Мандельброта в физике) экспоненциального } \\
\text { роста или гибели, относительно которого } \\
\text { Лаплас создал методологию операторного } \\
\text { исчисления }\end{array}$ \\
\hline $\begin{array}{l}y=a x^{b} \exp (-c x)-\text { биотехнический закон в } \\
\text { упрощенной форме (П.М. Мазуркин), когда } \\
\text { показательный рост постепенно получает } \\
\text { экспоненциальное торможение }\end{array}$ & $\begin{array}{l}y=a \exp \left( \pm c x^{d}\right)-\text { закон экспоненциального } \\
\text { роста или гибели в полной форме (конструк- } \\
\text { ции), который имеет интенсивность, не рав- } \\
\text { ную единице (П.М. Мазуркин) }\end{array}$ \\
\hline & 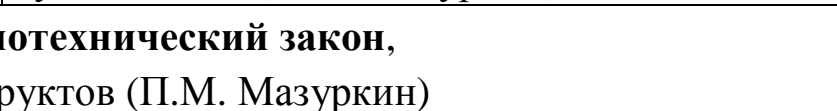 \\
\hline
\end{tabular}

Инварианты колебательных возмущений в виде асимметричных вейвлетсигналов также включают в себя конструкты из таблицы 1 как амплитуда (половина) и полупериод. Из-за асимметричности к вейвлетам принимается допущение о примерном равенстве нулю площади графиков над и под осью абсцисс.

В таблице 1 показаны наиболее встречающиеся инварианты (фрагменты). У них впереди могут быть расположены оперативные константы «+» или «-».

Шесть устойчивых законов распределения являются частными случаями биотехнического закона, показанного внизу таблицы 1. В названии закона слово «биотехнический» означает, что мы придерживаемся идей В. И. Вернадского о космической функции жизни.

Если известна эвристическая предыстория формирования числового поля (табличной модели), то вполне возможна смысловая расшифровка каждого вейвлет-сигнала. При этом вейвлет (волновая функция) в своей конструкции содержит те или иные математические инварианты из таблицы 1. Поэтому волновые функции могут быть разной конструкции по половине амплитуды и полупериоду колебания. В общем случае получаем одну обобщенную формулу.

Это обобщение опирается на биотехнический закон (см. табл. 1). Все известные законы распределения являются частными случаями биотехнического 
закона, что показано в учебных пособиях $[5,6]$.

Например, закон Гаусса (так называемого «нормального» распределения) запишется из закона экспоненциальной (по основанию с числом времени $e$ ) гибели по таблице 1 с дополнением четвертым параметром $f$ в виде формулы

$$
y=a \exp \left(-c(x-f)^{d=2}\right), b=0, f=\tilde{x},
$$

где $\tilde{x}-$ значение влияющей переменной при максимуме зависимого фактора.

Устойчивые законы и закономерности на их основе делают выбор уравнения для идентификации на статистических данных (числовых полях) осмысленным, и вероятностное моделирование остается только при поиске программной средой CurveExpert-1.40 значений параметров у искомой модели. Поэтому из теории идентификации первый этап (выбор случайной структуры уравнения) исключается и остается второй этап - случайная идентификация значений параметров искомой модели.

Идентификация структуры и параметров статистической модели проводится обработкой исходных $x$ и $y$ данных так:

- вначале выявляются детерминированные закономерности (тренды) как во времени (динамика), так и в срезе времени (состояние);

- затем эти трендовые закономерности дополняются колебательными возмущениями, идентифицируемых в виде асимметричных вейвлетов.

Идентификация параметрическая. Она выполнялась нами в программной среде CurveExpert-1.40 и нашей информационной технологией идентификации пользуются студенты (будущие бакалавры и магистры), а также аспиранты и докторанты. На многих примерах моделирования методология структурно-параметрической идентификации подробно показана в [2-10].

Выбор структуры искомой модели, которая является единственным алгебраическим решением по Декарту для неизвестной первообразной по волновым уравнениям, имеющим переменные амплитуды и период колебательного возмущения объекта исследования, выполняется из инвариантов в таблице 1. Процесс параметрической идентификации автоматически прекращается по условию достижения у параметров модели минимального приращения и останавливается 
пользователем при достижении конструируемой моделью погрешности измерений для заданного числового поля (табличной модели).

Поэтому потребность в наращивании модели новыми составляющими зависит от погрешности измерений при экспериментах. В связи с этим автору исходных данных всегда нужно оценивать погрешность измерений экспериментов и правильно записывать числа в таблице исходных данных.

Уровни адекватности закономерностей. В таблице 2 приведены интервалы изменения коэффициента корреляции как меры адекватности модели.

Уровни тесноты факторных связей

Таблица 2

\begin{tabular}{|c|c|c|c|c|}
\hline \multirow[b]{2}{*}{$\begin{array}{c}\text { Интервал ко- } \\
\text { эффициента } \\
\text { корреляции }\end{array}$} & \multicolumn{4}{|c|}{ Характер тесноты связи между факторами } \\
\hline & $\begin{array}{l}\text { существующая } \\
\text { классификация }\end{array}$ & $\begin{array}{c}\text { шкала для } \\
\text { технических } \\
\text { измерений }\end{array}$ & $\begin{array}{c}\text { шкала для } \\
\text { прецизионных } \\
\text { измерений } \\
\end{array}$ & $\begin{array}{c}\text { шкала для генной } \\
\text { инженерии и рядов } \\
\text { целых простых чисел }\end{array}$ \\
\hline 1 & \multirow{6}{*}{ сильная связь } & однозначная & однозначная & однозначная \\
\hline $0.999 \ldots 1.0000$ & & \multirow{4}{*}{ сильнейшая } & \multirow{2}{*}{ почти однозначная } & почти однозначная \\
\hline $0,99 \ldots 1,000$ & & & & чрезвычайно сильная \\
\hline $0,95 \ldots 0,99$ & & & сверхсильная & сверхсильная \\
\hline $0,90 \ldots 0,95$ & & & сильнейшая & сильнейшая \\
\hline $0,7 \ldots 0,9$ & & сильная & сильная & сильная \\
\hline $0,5 \ldots 0,7$ & \multirow{2}{*}{ слабая связь } & средняя & средняя & средняя \\
\hline $0,3 \ldots 0,5$ & & слабоватая & слабоватая & слабоватая \\
\hline $0,1 \ldots 0,3$ & \multirow{3}{*}{ нет связи } & слабая & слабая & слабая \\
\hline $0,0 \ldots 0,1$ & & слабейшая & слабейшая & слабейшая \\
\hline 0 & & нет связи & нет связи & нет связи \\
\hline
\end{tabular}

Существующая шкала квантификации тесноты связи между факторами (нет связи, слабая и сильная связь) является грубой и даже примитивной на современном уровне развития информатики и информационных технологий.

Нами было доказано, что именно анализ слабых связей, отбрасываемых при линейном моделировании и применении среднеарифметической факторной связи по закону Гаусса-Лапласа (так называемого «нормального» распределения), дает новые научные идеи. Эти идеи преобразуются в научно-технические решения, часть из которых оформляется в виде заявок на предполагаемые изобретения. За 14 лет было получено более 130 патентов на изобретения.

При анализе литературы по математической статистике было выявлено, что в аппроксимации сплошь и рядом применяется только линейная модель или 
же не имеющий физического смысла алгебраический полином. Это происходит из-за того свойства, что линейная модель инвариантна к любому типу распределения, в том числе и явно негауссовой структуры, и даже к явно скедастическим распределениям с переменной дисперсией при изменении объясняющей переменной. В связи с этим, если по сущности факторной связи заранее известно наличие двух членов в искомой модели, вначале всегда идентифицируется линейная модель из стандартной библиотеки. А затем проводится наращивание конструкции до биотехнического закона. Таким образом, известная линейная модель является только частным случаем, причем явно с низким уровнем адекватности при изучении колебательных возмущений в поведении объекта.

Из-за высокой грубости существующей классификации уровня адекватности нами была предложена для технических экспериментов, в которых погрешность измерений не превышает 5\%, другая шкала (третий столбец таблицы 2).

Однако выяснилось, что этой шкалы уровней адекватности также недостаточно из-за существования более точных табличных моделей. Для многих природных (биологических) объектов и результатов прецизионных физических измерений пришлось ввести еще два интервала уровня адекватности по четвертому столбцу таблицы 2. В пятом столбце таблицы 2 показана шкала квантификации тесноты связи между факторами для генов и рядов целых простых чисел.

Концепция моделирования по выборкам. Статистическая выборка - это многофакторное числовое поле, оформленное в виде табличной модели для последующей идентификации искомых трендов и волновых закономерностей.

Этим определением она существенно дополняется по сравнению с таблицами статистических изысканий, но, с другой стороны, для моделирования применяются только числовые выборки (клетки таблицы со словесными данными исключаются). Причем необязательно все клетки таблицы должны быть заполненными числами, но такая табличная модель усложняет процесс переноса данных в виде файлов данных в программную среду CurveExpert-1.40.

Поэтому в добротной табличной модели клетки должны иметь количественные и однородные (по сути) по принятому множеству факторов значения. 
При этом табличная модель необязательно имеет априорные эвристические пояснения. Как правило, авторы измерений, приводя в своих публикациях таблицы данных, как правило, дают неверные содержательные толкования. Статистическое моделирование позволяет исправить или углубить понимание табличной модели на основе получения апостериорной информации.

Феномен формализации состоит в том, что таблица результатов измерений, даже если составлена правильно, не может быть содержательно осмыслена без проведения факторного анализа с моделированием математических связей между факторами идентификацией бинарных отношений.

Тогда первичной становится табличная модель (исходное числовое поле), которая оценивается по погрешности измерений, а вторичным - искомое сложное алгебраическое уравнение (в смысле Декарта), составленное из инвариантов таблицы 1 (в смысле кирпичиков Гильберта).

Этот процесс идентификации есть статистическая идентификация.

Сама первообразная в виде неизвестного интегрального уравнения становится не нужным, хотя, может быть, кто-то и сумеет получать интегралы по нашим моделям. Это было бы великим научным созиданием, по значимости не меньшим общего уравнения Максвелла для электромагнетизма.

Детерминированная модель. Вначале выявляют не волновые модели.

В общем случае не волновая детерминированная модель (например, тренд или тенденция) содержит сумму двух биотехнических законов в виде

$$
y_{m}=y_{m 1}+y_{m 2}, y_{m 1}=a_{1} x^{a_{2}} \exp \left(-a_{3} x^{a_{4}}\right), y_{m 2}=a_{5} x^{a_{6}} \exp \left(-a_{7} x^{a_{8}}\right),
$$

где $y_{m}$ - тренд (тенденция), $x$ - объясняющая переменная, $a_{1} \ldots a_{8}$ - параметры обобщенной модели (2).

При этом каждый параметр модели (2) имеет физический смысл. А понятие «тренд» нами распространяется не только на динамические ряды (время является объясняющей переменной), но и на другие виды рядов распределений, появляющиеся в срезе времени как физические состояния.

Не волновой характер общая модель (2) получает в двух случаях:

1) когда шаг дискретности измерений слишком большой по сравнению с 
периодом колебательного возмущения реального процесса (например, импульс электрокардиограммы требует регистрации амплитуды через каждые 0,001 с);

2) когда интервал процесса измерений мал по сравнению с полупериодом колебания измеряемого показателя (динамика глобальной среднегодовой температуры в точке Земли требует регистрации за 1000 лет и более).

Тогда тренд появляется из-за несоразмерности разных шкал времени.

Асимметричный вейвлет. Мы придерживаемся концепции Декарта о необходимости применении алгебраического уравнения общего вида напрямую как конечного математического решения неизвестных интегральных уравнений. Для обобщения был предложен новый класс волновых функций [2-10].

Условиям физического существования наиболее полно удовлетворяет асимметричная вейвлет-функция вида

$$
\begin{gathered}
y=\sum_{i=1}^{m} y_{i}, \\
y_{i}=a_{1 i} x^{a_{2 i}} \exp \left(-a_{3 i} x^{a_{4 i}}\right) \cos \left(\pi x /\left(a_{5 i}+a_{6 i} x^{a_{7 i}} \exp \left(-a_{8 i} x^{a_{9 i}}\right)-a_{10 i}\right),\right.
\end{gathered}
$$

где $y$ - показатель (зависимый фактор), $i$ - номер составляющей модели (3), $m$ - количество членов в модели (3), причем косинус является связующим звеном между геометрией и алгеброй, $x$ - объясняющая переменная (влияющий фактор), $a_{1} \ldots a_{10}$ - параметры, принимающие числовые значения в ходе структурно-параметрической идентификации формулы (3) по мере увеличения коэффициента корреляции в ходе процесса идентификации.

Указанное уравнение, из-за высокой погрешности измерений в разных областях науки, встречается редко, хотя будущее, при изменении методики и повышении точности эволюционного эксперимента, несомненно, за формулой (3).

В большинстве случаев прошлых и настоящих экспериментов для идентификации закономерностей достаточна усеченная, по вложенной в тригонометрическую функцию формуле частоты, конструкция асимметричного вейвлета

$$
y=\sum_{i=1}^{m} y_{i}, y_{i}=a_{1 i} x^{a_{2 i}} \exp \left(-a_{3 i} x^{a_{4 i}}\right) \cos \left(\pi x /\left(a_{5 i}+a_{6 i} x^{a_{7 i}}\right)-a_{8 i}\right) .
$$

При этом количество $m$ членов в наших примерах достигала до 120 и бо- 
лее. Например, для идентификации изменения относительного атомного веса химических элементов в зависимости от порядкового номера в периодической таблице Д. И. Менделеева было получено 91 составляющая, из которых 89 членов являются асимметричными вейвлетами. Для описания волновой адаптации биологических объектов к окружающей среде были получена статистическая модель с 120 членами. Чем выше точность измерений, тем большее количество членов дает методология вейвлет-анализа на основе уравнения (4).

Как правило, общая стохастическая волновая функция (4), в которой не волновые части (2) становятся частными случаями и показывают детерминированное на интервале времени измерений поведение объекта исследования.

Это позволяет идентифицировать составной статистической моделью поведение многих астрономических и физических, биологических и экологических, социально-экономических и иных объектов и их систем.

Динамический ряд как череда сигналов. Физико-математический подход предполагает понимание смысла динамического ряда как отражения какого-то составного процесса или же множества реальных последовательно и параллельно происходящих природных или иных процессов. Впервые удалось получать модели многих типов рядов динамики на концепции аддитивного разложения любого динамического ряда на множество сигналов.

Сигнал - это материальный носитель информации. А информация нами понимается как мера взаимодействия. Сигнал может генерироваться, но его приём не обязателен. Так, например, ряд простых чисел известен несколько тысяч лет, но суть его как множества сигналов до сих пор не была раскрыта. Аналогично многие природные явления и процессы на эвристическом уровне науке давно понятны, но количественно они до сих пор не расшифрованы.

Например, это относятся к ряду чисел Вольфа солнечной активности, кривой Киллинга по концентрации окиси углерода в атмосфере Земли в разных точках, кривой Кузнеца для экономических процессов.

По состоянию на середину 1980-х годов выявлено 1380 разновидностей экономических циклов продолжительностью от 20 часов до 700 лет. Поэтому 
мы считаем, что все они являются частными случаями экономических колебаний с переменной частотой или в уравнении (2.4) с переменным полупериодом. Если полвека назад можно было на короткий промежуток времени говорить о колебаниях с постоянным периодом и пытаться классифицировать их по периодичности, то в начале XXI века периодичность колебаний поведения социально-экономических систем стала сильноизменчивой.

Экономические колебания возникают из-за трех основных причин:

1) колебательная адаптация к окружающей среде является важнейшим свойством любой природы (живого и косного вещества по В.И. Вернадскому), поэтому не исключение и экономические системы;

2) субъектное представление как экономического ресурса любых природных и природно-техногенных объектов (техникой как поведенческой структурой владеют не только люди), как правило, приводит к неосознанному поведению лиц принимающих решения, что сильно увеличивает риск возрастания амплитуды и снижения периода колебательной адаптации к своему окружению управляемой этими лицами социально-экономической системы;

3) с ростом численности человечества возрастает и количество социальноэкономических систем, а также и их поведенческих структур, поэтому неосознанное колебание каждого из этих систем приводит к наложению с колебаниями других, что при диаметрально противоположных политических интересах и мотивах приводит к возникновению глобальных экономических кризисов.

Сигналом может быть любой физический процесс или его часть. Получается, что изменение множества непонятных современной науке сигналов давно известно, например, через ряды гидрометеорологических измерений во многих точках планеты. Очень много несознаваемых нынешней наукой сигналов возникает из-за отсутствия статистического текущего моделирования экономического собственного поведения внутри каждой социально-экономической системы или её составной части (элемента-института этой структуры).

Если признать любое измеренное поведение как изучаемый сигнал, то любое уравнение типа (4) можем записать как вейвлет-сигнал вида 


$$
y_{i}=A_{i} \cos \left(\pi x / p_{i}-a_{8 i}\right), A_{i}=a_{1 i} x^{a_{2 i}} \exp \left(-a_{3 i} x^{a_{4 i}}\right), p_{i}=a_{5 i}+a_{6 i} x^{a_{7 i}},
$$

где $A_{i}$ - амплитуда (половина) вейвлета (ось $y$ ),

$$
p_{i} \text { - полупериод колебания (ось } x \text { ). }
$$

По формуле (5) с двумя фундаментальными физическими постоянными $e$ (число Непера или число времени) и $\pi$ (число Архимеда или число пространства) образуется изнутри изучаемого явления и/или процесса квантованный асимметричный вейвлет-сигнал. Тогда для конкретной социальноэкономической или иной эргатической системы встает двоякая задача:

вначале выявить закономерности собственного поведения; затем выявить закономерности поведение внешней среды; затем уточнить на будущее сценарии собственного поведения;

после этого итерационно двигаться по осознаваемой и управляемой траектории колебательной адаптации значениями параметров к внешней среде.

Понятие вейвлет-сигнала позволяет абстрагироваться от физического смысла самих рядов (в общем случае не только динамических) и рассматривать их аддитивное разложение.

Заключение. В предложенной методологии идентификации обобщенной биотехнической функции через несколько столетий восстанавливается универсальный метод Рене Декарта, выполняемый по следующей схеме:

1) задача функционирования любого вида сводится к математической задаче статистического моделирования (части математического моделирования);

2) математическая задача моделирования сводится к алгебраической задаче, составленной из формул устойчивых законов и закономерностей;

3) любая алгебраическая задача сводится к решению одного уравнения.

Это единственное уравнение записывается в виде асимметричного вейвлет-сигнала (5) с переменными амплитудой и периодом.

Ключом к составлению конструкции этого алгебраического уравнения является биотехнический закон. Он отображает совместное и одновременное действие двух сил. Причем в большинстве случаев действие реализуется устойчивым показательным законом роста, а противодействие выражается устойчивым 
экспоненциальным законом гибели.

Исходное статистическое уравнение составляется исходя из априорных эвристических представлений об изучаемом явлении или процессе. Причем биотехнический закон безоговорочно исходит из первичности живого вещества над косным веществом по теории В.И. Вернадского.

В ходе структурно-параметрической идентификации и повторных сеансов параметрической идентификации существенное приращение новой информации об изучаемом явлении или процессе появляется при дополнении основного тренда дополнительными волновыми составляющими.

Как убежденно доказывал Д. Пойа ${ }^{1}$, в процессе решения алгебраических уравнений появляются своеобразные математические открытия и на этой основе - принципиально новые технические решения на уровне изобретений ${ }^{2,3,4}$.

Иерархия методов статистической идентификации следующая:

1) формирование табличной модели и кластерный анализ факторов;

2) рейтинг объектов и субъектов в заданной системе;

3) ранговые распределения значений факторов;

4) волновая адаптация параметров системы;

5) факторный анализ показателей системы;

6) рейтинг влияющих и зависимых факторов;

7) вейвлет-анализ отношений между факторами;

8) фрактальный анализ вейвлетов;

9) составление прогнозных моделей по вейвлетам динамики;

10) многофакторное иерархическое моделирование.

В последующих статьях будут показаны примеры идентификации и факторного анализа параметров структуры и поведения различных объектов.

\footnotetext{
${ }^{1}$ Пойа Д. Математическое открытие. Решение задач: Основные понятия, изучение и преподавание. М.: Наука, 1976. 448 с.

${ }^{2}$ Мазуркин П.М. Самоорганизация студента в инновационном обучении и изобретательской деятельности: науч.-учеб. изд. Йошкар-Ола: МарГТУ, 2011. 255 с.

${ }^{3}$ Мазуркин П.М. Единство материального и духовного мира в научно-техническом тврчестве // Современные проблемы науки и образования. 2010. № 3. С.107-129.

${ }^{4}$ Мазуркин П.М. Психолого-педагогические основы научно-технического творчества // Современные проблемы науки и образования. 2010. № 3. С.58-70.
} 


\section{Список литературы:}

1. Леонтьев В.Л. Экономическое эссе. Теории исследования, факты и политика: Пер. с англ. М. Политиздат, 1990. 415 с.

2. Мазуркин П.М. Статистическая социология: учеб. пос. Йошкар-Ола: МарГТУ, 2005. 182 с.

3. Мазуркин П.М. Статистическая экология: учеб. пос. Йошкар-Ола: МарГТУ, 2004. 308 c.

4. Мазуркин П.М. Статистическая эконометрика: учеб. пос. Йошкар-Ола: МарГТУ, 2006. 376 с.

5. Мазуркин П.М., Порядина О.В. Эконометрическое моделирование: практикум. Йошкар-Ола: МарГТУ, 2009. 204 с.

6. Мазуркин П.М., Филонов А.С. Математическое моделирование. Идентификация однофакторных статистических закономерностей: учеб. пос. Йошкар-Ола: МарГТУ, 2006. 326 с.

7. Mazurkin P.M. Invariants of the Hilbert Transform for 23-Hilbert Problem. Advances in Sciences and Humanities, 2015, Vol. 1, No. 1, 1-12, doi: 10.11648/j.ash.20150101.11.

8. Mazurkin P.M. Method of Identification of Wave Regularities According to Statistical Data (Of Dynamics of a Rate of Inflation of US Dollar). Advances in Sciences and Humanitie. 2015, Vol. 1, No. 2, 45-51, doi: 10.11648/j.ash.20150102.12.

9. Mazurkin P.M. Wavelet Analysis Statistical Data. Advances in Sciences and Humanities. 2015, Vol. 1, No. 2, 30-44, doi: 10.11648/j.ash.20150102.11.

10. Mazurkin P.M.. Statistical modeling of entire prime numbers / International Journal of Engineering and Technical Research (IJETR) ISSN: 2321-0869. Volume2. Issue-8. August 2014. P.148-158. 